\title{
A study on Histomorphological Spectrum of Nasal Polyp
}

\author{
K Pushpalatha*, Sreedevi CH, Soujanya R \\ Department of Pathology, Maheshwara Medical College, Sangareddy, India
}

\begin{abstract}
Background: Nasal Polyp is commonly encountered in clinical practice and important from clinical and pathological perspectives as they have varieties of histological patterns. The Polypoidal masses in nasal cavity form a complex group of lesions with wide spectrum of histopathological features, mainly grouped under allergic and inflammatory. Histopathological examination shows a spectrum of lesions ranging from the non-neoplastic ones to neoplastic tumors.

Methods: Present study included 153 polypoidal lesions of the nasal cavity during a period of one year. All the tissues were fixed in $10 \%$ buffered formalin, processed, stained with $\mathrm{H} \& \mathrm{E}$ and studied for various histopathological patterns. Periodic acid Schiff's and reticulin stains were used wherever necessary.

Results: classifying the sinonasal lesions according to histo-pathological features into various types helps us to know the clinical presentation, treatment, clinical outcome and prognosis of the disease. Although most of nasal polyps sent for histopathology are inflammatory, secondary to infection or allergy, various benign and malignant lesions of nose may present as polypoidal masses,
\end{abstract}

Conclusion: The study recommends, all polyps need histo-pathological examination.

Keywords: Nasal Polyps, Sinonasal Lesions, Histo-Pathology.

\section{Introduction}

Sinonasal lesions are the common lesion encountered in clinical practice and important from clinical and pathological perspectives as they give rise to varieties of histological patterns and grades of malignancies. ${ }^{[1]}$ The presence of mass in the nose is a seemingly simple problem; however, it raises numerous questions about differential diagnosis. Although neoplasms of the nose and paranasal sinuses are not common, they are of interest because of their various types. It has been found, the nose and paranasal sinuses account for less than $1 \%$ of all malignant tumors in general, not more than $3 \%$ of the head and neck region malignancies. Clinically sometimes, it becomes quite impossible to distinguish between inflammatory conditions presenting as simple polyps, polypoidal lesions due to specific disease and polypoid neoplasm (benign and malignant). Therefore it becomes important that all polyps and polypoidal lesions should be submitted for histopathological examination.

Nasal polyps are defined as prolapsed lining of the nasal sinuses. They are essentially rounded projections of edematous membrane. They are often bilateral and multiple which lead to visible broadening of nose. The commonest site of origin is in the ethmoidal labyrinths, particularly from the mucosa of middle turbinate. In nasal cavity, tumors of various type have a tendency to become polypoid. Thus an epithelial papilloma of the nasal cavity often resembles a nasal polyp. ${ }^{[5]}$ Some lesions are specific to certain location, for e.g., epithelial papilloma of turbinate, juvenile angiofibroma of nasopharynx. Polypoidal masses of the nasal cavity form complex group lesions with a wide spectrum of histopathological features. ${ }^{[6]}$ The true nasal polyps are the tumor like nonneoplastic polypoid masses arising from nasal cavity and sinuses, classified interms of Allergic nasal polyp, which constitutes 85-90\%, Fibroinflammatory polyp characterized by chronic inflammation and metaplastic changesof the overlying epithelium. Other variants present with pronounced hyperplasia of seromucinous glands and Polyp with atypical stroma, rarely seen. The current study initiated to determine the Hisotopathological variations to categorize nasal polyps which are difficult to identify by clinical findings.

\section{Material and Methods}

A total number of 153 Specimens received in the histopathology section of the Dept. of Pathology of our institution with the clinical diagnoses of 'Nasalpolyp'. The age and sex of the patients were recorded. The tissues were routinely processed for histopathological sections are made $4-6 \mathrm{~mm}$ on a rotatory microtome and were stained by H\&E stain. Special stains by Reticulin and PAS methods were undertaken wherever applicable. The cases were classified into Nonneoplastic, Benign Neoplastic lesions 
and Malignant Neoplastic lesions. The Neoplastic lesions were further classified according to WHO classification on histopathological examination.

Data Analysis: Data was analyzed by SPSS 20.0. Data was expressed as mean $\pm \mathrm{SD}$.

\section{Results}

Among Non-neoplastic lesions $(\mathrm{n}=98)$, allergic Nasal polyp cases were high with $56.12 \%$ (table 1). Among

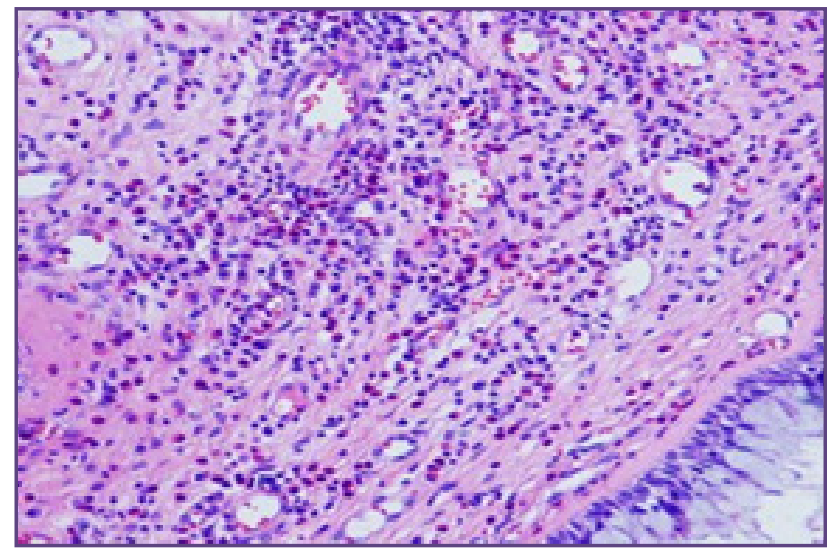

Fig. 1: Illustrating Allergic nasal polyp. benign neoplastic cases $(\mathrm{n}=44)$ distribution, Adenomatous polyps are high with $40.91 \%$ (table 2 ). Among distribution in malignant neoplastic lesions, Squamous cell carcinoma is highest with $54.55 \%$ (table 3 ). Figure 2 presents allergic nasal polyp and Figure 1-3 shows the various Allergic nasal polyp inflammatory nasal polyp and Haemangioma.

\section{Discussion}

Though nasal polyps are affecting about four percent of the population, their etiology is still not clear. ${ }^{[16]}$ Polypoidal

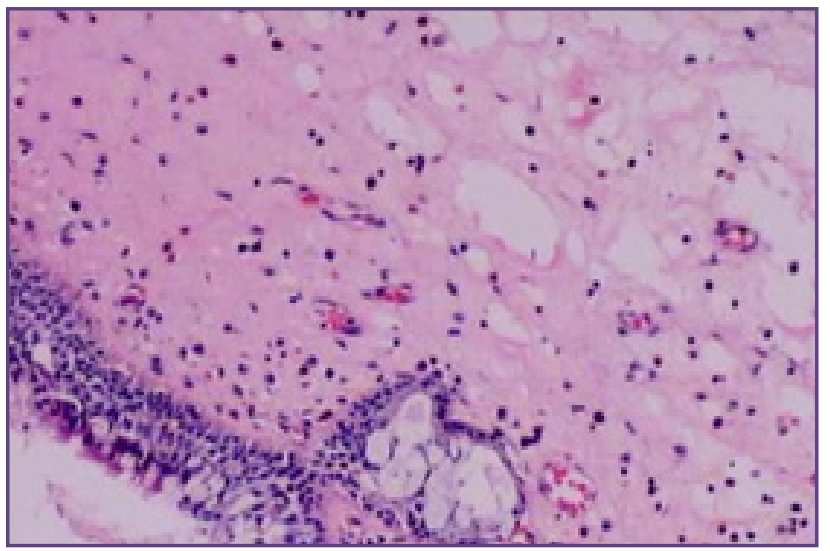

Fig. 2: Illustrating Inflammatory nasal polyp

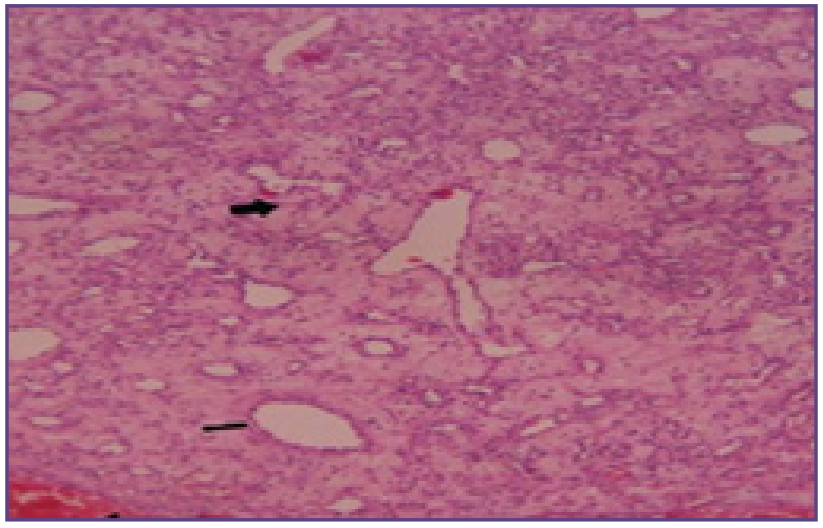

Fig. 3: Haemangioma.

Table 1: Non-neoplastic lesions $(n=98)$

\begin{tabular}{|l|c|c|c|c|}
\hline Diagnosis & No of cases & Male & Female & $\%$ \\
\hline Nasal polyp-Allergic & 55 & 43 & 12 & $56.12 \%$ \\
\hline Nasal polyp-inflammatory & 30 & 23 & 7 & $30.61 \%$ \\
\hline Rhnosporidiosis & 9 & 6 & 3 & $9.18 \%$ \\
\hline Rhino scleroma & 0 & 0 & 0 & $0.00 \%$ \\
\hline Mucocele & 1 & 1 & 0 & $1.02 \%$ \\
\hline Nasoalvelor cyst & 0 & 0 & 0 & $0.00 \%$ \\
\hline Tuberculous granuloma & 1 & 1 & 0 & $1.02 \%$ \\
\hline Fungal granuloma & 2 & 1 & 1 & $2.04 \%$ \\
\hline TOTAL & $\mathbf{9 8}$ & $\mathbf{7 5}$ & $\mathbf{2 3}$ & \\
\hline
\end{tabular}

Data was expressed as frequency and percentages. 
Table 2: Benign neoplastic lesions $(n=44)$.

\begin{tabular}{|c|c|c|c|c|c|}
\hline \multicolumn{2}{|l|}{ Diagnosis } & \multirow{2}{*}{$\begin{array}{c}\text { No of cases } \\
4\end{array}$} & \multirow{2}{*}{$\frac{\text { Male }}{3}$} & \multirow{2}{*}{$\begin{array}{c}\text { Female } \\
1 \\
\end{array}$} & \multirow{2}{*}{$\begin{array}{c}\% \\
9.09 \% \\
\end{array}$} \\
\hline & Inverted papilloma & & & & \\
\hline Epithelial & Adenomatous polyps & 18 & 14 & 4 & $40.91 \%$ \\
\hline & Squamous papilloma & 2 & 1 & 1 & $4.55 \%$ \\
\hline \multirow{7}{*}{ Mesenchymal } & Haemangioma & 14 & 9 & 5 & $31.82 \%$ \\
\hline & Angiofibroma & 4 & 3 & 1 & $9.09 \%$ \\
\hline & Haemangiopericytoma & 1 & 1 & 0 & $2.27 \%$ \\
\hline & Fibroma & 0 & 0 & 0 & $0.00 \%$ \\
\hline & Schwannoma & 0 & 0 & 0 & $0.00 \%$ \\
\hline & Osteiod osteoma & 0 & 0 & 0 & $0.00 \%$ \\
\hline & Leiomyoma & 1 & 1 & 0 & $2.27 \%$ \\
\hline TOTAL & & 44 & 32 & 12 & \\
\hline
\end{tabular}

Data was expressed as frequency and percentages.

Table 3: Malignant neoplastic lesions $(n=11)$.

\begin{tabular}{|l|l|c|c|c|c|}
\hline \multicolumn{2}{|l|}{ Diagnosis } & No of cases & Male & Female & \% \\
\hline Epithelial & Squamous cell carcinoma & 6 & 5 & 1 & $54.55 \%$ \\
\hline & Adenocarcinoma & 3 & 2 & 1 & $27.27 \%$ \\
\hline & Adeniodcysticarcinoma & 0 & 0 & 0 & $0.00 \%$ \\
\hline & Malignant Melanoma & 1 & 1 & 0 & $9.09 \%$ \\
\hline & Mucoepidermoid carcinoma & 0 & 0 & 0 & $0.00 \%$ \\
\hline Mesenchymal & Neuroblastoma & 0 & 0 & 0 & $0.00 \%$ \\
\hline & NonHodgkins lymphoma & 1 & 1 & 0 & $9.09 \%$ \\
\hline TOTAL & Angiosarcoma & 0 & 0 & 0 & $0.00 \%$ \\
\hline
\end{tabular}

Data was expressed as frequency and percentages.

masses in the nasal cavity form a complex group of lesions with a wide spectrum of histopathological features. ${ }^{[6]}$ While there are many nonneoplastic lesions including mainly the allergic and inflammatory one, there are also good number of neoplastic tumefaction in the nose and nasal sinuses. These lesions are often quite impossible to distinguish clinically and are labelled as nasal polyp. ${ }^{[7]}$ Histopathological examination of such polypoidal masses show a spectrum of lesions ranging from nonneoplastic ones to neoplastic tumors including benign and malignant neoplasms. ${ }^{[8]}$ The true nasal polyps are the tumor like nonneoplastic polypoidal masses arising from nasal cavity and sinuses. Two types are encountered- one is associated with nasal allergy and another with numerous inflammatory or granulomatous polyp. ${ }^{[9]}$

In our study, we have observed 98 cases were Nonneoplastic lesions, 44 cases were benign neoplastic lesions and 11 cases were malignant neoplastic lesions. Nasal polyp with $56.12 \%$ found to be highest in non-neoplastic lesions, followed by Nasal polyp inflammatory lesions with $30.61 \%$. These polyps are found to be common in male. In benign neoplastic cases, Adenomatous polyps with $40.91 \%$ found to highest, followed by Haemangioma with $31.82 \%$. These type of polyps also mostly found in male.In malignant neoplastic cases, Squamous cell carcinoma with $54.55 \%$ found to be highest, followed by Adenocarcinoma with $27.27 \%$. The incidence of nasal polyps was slightly higher in this study $(82.06 \%)$ compared to the observations by Tondon et al (64\%) and Anjali et al (62.85\%). The age range of the patients was from 10 to 80 years. ${ }^{[10]}$ Most commonly patients are in $2^{\text {nd }}$ to $3^{\text {rd }}$ decade which is comparable with Ghosh and Bhattacharya and Zafar et al. ${ }^{[1]}$

There is male preponderance with male to female ratio of 1.53:1 which was same as that observed by Zafar et al and Dasgupta et al. Although adolescence or early childhood is stated to be the commonest age of occurrence, there are reports of this disease occurring in all age groups. ${ }^{[12-}$ ${ }^{14]}$ Nasal polyps were bilateral in $60 \%$ cases in our study, while according to Batsakis bilateralism was the rule. In 
our study, 2 cases $(4.36 \%)$ of fungal infection were found in the age group of 20-60 years with male predominance which is comparable with Ghosh and Bhattacharya. ${ }^{[15]}$ Hemangiopericytoma is a rare angiogenic tumor accounting for only $1 \%$ of total cases in our study. As the initiating cause of the nasal polyps are still not clear, it is essential to understand the Histomorphological Spectrum of Nasal Polyp. Our study attempted to add further knowledge about etiology of nasal polyps.

\section{Conclusions}

To conclude, classifying the sinonasal lesions according to histopathological features into various types helps us to know the clinical presentation, treatment, clinical outcome and prognosis of the disease. Although most of nasal polyps sent for histopathology are inflammatory, secondary to infection or allergy, various benign and malignant lesions of nose may present as polypoidal masses, so all polyps need histopathological examination.

\section{Acknowledgements}

We would like to acknowledge the staff of departments of both Pathology for extending their support and cooperation to carry out the study.

\section{References}

1. Cotran RS, Kumar V, Stanly L. Robins Pathologic basis of disease, 6th edn. W.B. Saunders Company, 2000.

2. Kalpana Kumari MK, Mahadeva KC. Polypoidal lesions in the nasal cavity. J Clin Diagn Res. 2013; 7(6):1040-42.

3. Drake Lee AB. Nasal polyps. Chapter 10 in Scott Browns Otolaryngology Rhinology 6th edn, 1984.

4. Newton JR, Kim Wong Ah-See. A review of nasal polyposis. Ther Clin Risk Manag. 2008 Apr; 4(2): 507-512.

5. Bateman ND, Fahy C, Woolford TJ. Nasal polyps: still more questions than answers. J Laryngol Otol. 2003;117 :1-9.
6. Dalziel K, Stein K, Round A, et al. Systematic review of endoscopic sinus surgery for nasal polyps. Health Technol Assess. 2003;7:1-159.

7. Baudoin T, Kalogjera L, Hat JI. Capsaicin significantly reduces sinonasal polyps. Acta Otolaryngol. 2000;120 :307-11.

8. Luis CilloZenaida BA. "Cancer of Nasal Cavity" Cancer. 1976; 37; 1458-1463.

9. Dalziel K, Stein K, Round A, et al. Systematic review of endoscopic sinus surgery for nasal polyps. Health Technol Assess. 2003;7:1-159.

10. Dasgupta A, Ghosh RN, Mukherjee C. Nasal polyps histopathologic spectrum. Indian J Otolaryngol Head Neck Surg. 1997;49(1):32-37.

11. Jyothi AR et al. Morphological spectrum of lesions in the sinonasal region. Journal of evolution of medical and dental sciences. 2013;37(2);7175-86.

12. Khan N, Zafar U, Afroz N, Ahmad SS, Hasan A. Masses of nasal cavity, paranasal sinuses and nasopharynx: A clinicopathological study Indian J Otolaryngol Head Neck Surg. 2006 Jul;58(3):259-263

13. Modh S K, Delwadia K N, Gonsai R N. Histopathological spectrum of sinonasal masses- A study of 162 cases. Int J Cur Res Rev. 2013;5(3):83-9.1

14. Zafar U, Khan N, Afroz N et al.Clinicopathological study of nonneoplastic lesions of nasal cavity and paranasal sinuses. Indian J Pathol Microbiol. 2008;51(1):26-29.

15. Kulkarni AM, Mudholkar VG, Acharya AS, Ramteke RV. Histopathological study of lesions of nose and paranasal sinuses.Indian J Otolaryngol Head Neck Surg. 2012; 64(3):275-289.

16. Fokkens W, Lund V, Mullol J. European Position Paper on Rhinosinusitis and Nasal Polyps Group. EP3OS 2007: European position paper on rhinosinusitis and nasal polyps 2007. A summary for otorhinolaryngologists. Rhinology. 2007; 45:97-101.

*Corresponding author:

Dr K Pushpalatha, Associate Professor, Department of Pathology, Maheshwara Medical College, Sangareddy, India

Date of Submission : 21.11.2016

Date of Acceptance : 21.01.2017

Financial or other Competing Interests: None. 